\title{
On the Lattice Structure of Subsets of Octagonal Neighborhood Sequences in $\mathbb{Z}^{n}$
}

\author{
András Hajdu ${ }^{1}$ and Lajos Hajdu ${ }^{2, \star}$ \\ ${ }^{1}$ Faculty of Informatics, University of Debrecen, \\ P.O. Box 12, H-4010 Debrecen, Hungary \\ hajdua@inf .unideb.hu \\ ${ }^{2}$ Institute of Mathematics, University of Debrecen, \\ and the Number Theory Research Group of the Hungarian Academy of Sciences, \\ P.O. Box 12, H-4010 Debrecen, Hungary \\ hajdul@math.klte.hu
}

\begin{abstract}
In this paper we investigate the lattice properties of several special, but important subsets of $S_{n}$, the set of $n \mathrm{D}$ octagonal neighborhood sequences in $\mathbb{Z}^{n}$, with respect to two ordering relations $\sqsupseteq^{*}$ and $\sqsupseteq$. Both orderings have some natural meaning, especially $\beth^{*}$ compares the "speed" how neighborhood sequences spread in $\mathbb{Z}^{n}$. We summarize our and the previous related results in a table. In particular, our theorems can be considered as extensions of some results from [1, 2, 3.
\end{abstract}

\section{Introduction}

Motions in the digital space $\mathbb{Z}^{n}$ play an important role in several parts of discrete mathematics, including discrete geometry and digital image processing. The most important and most well-known motions in $\mathbb{Z}^{2}$ are the so-called cityblock (or von Neumann) and the chessboard (or Moore) motions. They are based upon the classical 4-neighborhood and 8-neighborhood relations, respectively. If we combine these relations in a strictly alternating way, then we get the so-called octagonal distance. These motions and the induced distance functions were (partly) introduced and extensively studied in the pioneer paper of Rosenfeld and Pfaltz [4]. Das, Chakrabarti and Chatterji 5] investigated periodic octagonal neighborhood sequences (i.e. arbitrary periodic combinations of the 4 - and 8 neighborhood relations). They performed similar investigations also in $\mathbb{Z}^{n}$. Such sequences are dealt with in many papers, see e.g. [1,6,5,7,8] and the references given there. Fazekas, Hajdu and Hajdu [2] extended the theory to the case of arbitrary octagonal neighborhood sequences, where any (not necessarily periodic) sequences are considered. Such sequences are much more appropriate for certain purposes. For example, the authors in [9] constructed digital metrics on $\mathbb{Z}^{2}$ based upon such sequences, which provide the best approximation to the Euclidean distance in a certain sense. Using periodic sequences, only some finite parts of such sequences can be obtained, see e.g. 6, 10.

\footnotetext{
* Research supported in part by the János Bolyai Research Fellowship of the Hungarian Academy of Sciences, and by the OTKA grants F043090, F034981 and T042985.
} 
In [2] Fazekas, Hajdu and Hajdu described the lattice structure of $S_{n}$, the set of (general) octagonal neighborhood sequences on $\mathbb{Z}^{n}$, with respect to two ordering relations $\sqsupseteq^{*}$ and $\sqsupseteq$. The structure of $P_{n}$, the subset of $S_{n}$ consisting of the periodic octagonal neighborhood sequences was also investigated here. Further, the same authors in [3] (among other results) described the lattice structure of $M_{n}$, i.e. the set of neighborhood sequences inside $S_{n}$ which generate metrics.

In this paper we investigate the lattice structures of some other subsets of $S_{n}$, as well. Our main motivation is to tie up some loose ends in this field, and also to extend existing results to other subsets, not examined so far. We start with $U_{n}$, the set of ultimately periodic octagonal neighborhood sequences. The study of such sequences is motivated by their importance, as they yield a natural extension of the periodic sequences. We also refer to the paper of Hajdu, Hajdu and Tijdeman [11] where such sequences are closely investigated, under more general circumstances. We also consider the so-called Lyndon sequences $L_{n}$. Such sequences play a central role in many problems of word theory, see e.g. the book [12. Finally, as a natural generalization of the sets $P_{n}, U_{n}, M_{n}$ we consider the set $D_{n}$ consisting of sequences $A$ such that all symbols in $A$ have densities. Beside these results we provide some theorems for $P_{n}$ and $M_{n}$, as well. The extension of the investigated families is meaningful in applications, e.g. for the $U_{n}$ sequences that are generated by finite data. The main interest of comparing the sequences lies in deciding about their relative spreading speed in $\mathbb{Z}^{n}$ (see also $[13]$ ).

The structure of the paper is as follows. In section 2 we introduce some concepts and notation. Section 3 contains our main results summarized in a table, as well. We give auxiliary results and prove our theorems in section 4 .

\section{Basic Concepts and Notation}

In this section we introduce the necessary notation. First we recall some standard notions from the theory of words; for more details see e.g. 12,14. Let $\Gamma$ be a finite alphabet. As usual, finite sequences consisting of symbols from $\Gamma$ are called $\Gamma$ words, or simply words. The concatenation $u v$ of two words $u$ and $v$ is understood in the well-known way, and by $|u|$ we denote the number of symbols in $u$. If $u$ is a word and $n \in \mathbb{N}$, then $u^{n}$ means the $n$-fold concatenation $u u \ldots u$. If $W$ is a (one-sided) infinite sequence of symbols from $\Gamma$, then we call $W$ a $\Gamma$-sequence, or simply a sequence. If $u$ is a word and $W$ is a sequence then the concatenation $u W$ is defined in the classical way, as well as the prefixes and suffixes of words and sequences. Finally, if $u$ is a word then $\bar{u}$ denotes the sequence $u u u \ldots$. .

Now we introduce some standard notation concerning neighborhood sequences, see e.g. [5, 2]. Let $m, n \in \mathbb{N}$ with $m \leq n$. The points $p=\left(p_{1}, \ldots, p_{n}\right)$, $q=\left(q_{1}, \ldots, q_{n}\right)$ in $\mathbb{Z}^{n}$ are $m$-neighbors, if the following conditions hold:

$-\left|p_{i}-q_{i}\right| \leq 1$, for all $1 \leq i \leq n$,

$-\sum_{i=1}^{n}\left|p_{i}-q_{i}\right| \leq m$. 
The sequence $A=(A(i))_{i=1}^{\infty}$, where $A(i) \in\{1, \ldots, n\}$ for all $i \in \mathbb{N}$, is called an $n$-dimensional (shortly $n \mathrm{D}$ ) neighborhood sequence. The set of such sequences is denoted by $S_{n}$. Obviously, $S_{n}$ can be considered as the set of $\Gamma$-sequences with $\Gamma=\{1, \ldots, n\}$. For $A \in S_{n}$ and $k \in\{1, \ldots, n\}, i, j \in \mathbb{N}$ with $i \leq j$ put

$$
\mathbf{k}(A, i)=\#\{A(l) \mid A(l)=k, l=1, \ldots, i\}
$$

and let

$$
\mathbf{k}(A, i, j)=\mathbf{k}(A, j)-\mathbf{k}(A, i) .
$$

The density $s_{k}$ of the symbol $k$ in $A$ is defined as

$$
s_{k}(A)=\lim _{i \rightarrow \infty} \frac{\mathbf{k}(A, i)}{i}, \text { for } k \in\{1, \ldots, n\},
$$

if the limit exists. We say that $A \in S_{n}$ have density, if $s_{k}(A)$ exists for every $k=1, \ldots, n$. In this case $s(A)=\left(s_{1}(A), \ldots, s_{n}(A)\right)$ is called the density tuple of $A$.

If for an $A \in S_{n}$ we have

$$
A=A(1) A(2) \ldots A(h) \overline{A(h+1) A(h+2) \ldots A(h+l)}
$$

for some $h, l \in \mathbb{N}$ then $A$ is called an ultimately periodic neighborhood sequence. In case of $h=0$, i.e. when $A=\overline{A(1) A(2) \ldots A(l)}, A$ is called periodic with period $l$. The set of periodic and ultimately periodic sequences is denoted by $P_{n}$ and $U_{n}$, respectively.

Let $p, q \in \mathbb{Z}^{n}$ and $A \in S_{n}$. The point sequence $p=p_{0}, p_{1}, \ldots, p_{t}=q$, where $p_{i-1}$ and $p_{i}$ are $A(i)$-neighbors in $\mathbb{Z}^{n}(1 \leq i \leq t)$, is called an $A$-path from $p$ to $q$ of length $t$. The $A$-distance $d(p, q ; A)$ of $p$ and $q$ is defined as the length of the shortest $A$-path(s) between them. As a brief notation, we also use $d(A)$ for the $A$-distance. If $d(A)$ is a metric on $\mathbb{Z}^{n}$, then $A$ is called a metrical neighborhood sequence. The set of such sequences is denoted by $M_{n}$.

A neighborhood sequence $A \in S_{n}$ is called a Lyndon sequence if for any (infinite) suffix $B$ of $A$, either $A=B$, or $A$ lexicographically precedes $B$. The set of such sequences is denoted by $L_{n}$. As it turns out, we have $M_{n} \subseteq L_{n}$. The literature of (finite) Lyndon words is very extensive; see e.g. [12. For some basic results and properties of Lyndon sequences see e.g. [15, 16].

In our structural investigations we examine some subsets of $S_{n}$ with respect to two partial orderings, $\sqsupseteq^{*}$ and $\sqsupseteq[1,2$. These orderings are defined in the following way. For $A, B \in S_{n}$ write $A \sqsupseteq^{*} B$ if and only if $d(p, q ; A) \leq d(p, q ; B)$ for every $p, q \in \mathbb{Z}^{n}$, and set $A \sqsupseteq B$ if and only if $A(i) \geq B(i)$ for every $i \in \mathbb{N}$. Obviously, $\sqsupseteq^{*}$ is a refinement of $\sqsupseteq$, that is $A \sqsupseteq B$ implies $A \sqsupseteq^{*} B$ for every $A, B \in S_{n}$. Further, from [2] we know that if $A, B \in S_{n}$ then $A \sqsupseteq^{*} B$ if and only if

$$
\sum_{i=1}^{j} \min \{k, A(i)\} \geq \sum_{i=1}^{j} \min \{k, B(i)\}, \text { for all } j \in \mathbb{N}, k=1, \ldots, n .
$$

We will use this property throughout the paper, without any further reference. 
We also note that it is obvious that $d(A)$ is not a metric on $\mathbb{Z}^{n}$ for every $A \in S_{n}$. Nagy [17]18] proved that $A \in M_{n}$ if and only if $B \sqsupseteq^{*} A$ for any (infinite) suffix $B$ of $A$. We will use this assertion without any further mentioning.

Now we recall a few basic concepts and facts from lattice theory. They also will be used throughout the paper without any further notice. Let $H$ be a partially ordered set. We say that $H$ is a lattice, if for any $A, B \in H$ the greatest lower bound $A \wedge B$ and the least upper bound $A \vee B$ of these elements exist. If for any $S \subseteq H$ the greatest lower bound $\bigwedge S$ and the least upper bound $\bigvee S$ of $S$ also exist, then the lattice $H$ is called complete. It is well-known that if $\bigwedge S$ exists for all subset $S$ of $H$, then $\bigvee S$ also exists for any subset, and vice versa. The lattice $H$ is distributive, if for any $A, B, C \in H$ we have

$$
(A \wedge B) \vee C=(A \vee C) \wedge(B \vee C) \text { and }(A \vee B) \wedge C=(A \wedge C) \vee(B \wedge C)
$$

In our investigations we consider greatest lower bounds and least upper bounds both with respect to $\sqsupseteq^{*}$ and $\sqsupseteq$. To make a distinction, the simple notation $\wedge$ and $\vee$ will refer to $\sqsupseteq$, while in case of $\beth^{*}$ we use $\wedge_{*}$ and $\vee_{*}$. In particular, a theorem from [2] guarantees that for any $A, B \in S_{2}$ we have

$$
\mathbf{2}\left(A \wedge_{*} B, i\right)=\min \{\mathbf{2}(A, i), \mathbf{2}(B, i)\}
$$

and

$$
\mathbf{2}\left(A \vee_{*} B, i\right)=\max \{\mathbf{2}(A, i), \mathbf{2}(B, i)\}
$$

for all $i \in \mathbb{N}$. A very important remark is that though we will work in several subsets of $S_{n}$, this notation will always refer to the corresponding upper or lower bounds in $S_{n}$.

\section{The Lattice Structure of Subsets of $S_{n}$}

In this section we give our results about the lattice structure of certain special, but important subsets of $S_{n}$. We investigate the lattice structures under both orderings $\sqsupseteq^{*}$ and $\sqsupseteq$. We start with noting that the lattice structure of $S_{n}$ under these orderings were clarified in [2]; see also Table[1. In particular, $\left(S_{2}, コ^{*}\right)$ and $\left(S_{n}, \sqsupseteq\right)$ for $n \geq 2$ are complete distributive lattices. However, $\left(S_{n}, コ^{*}\right)$ is not a lattice for $n \geq 3$.

\subsection{Periodic Neighborhood Sequences $-P_{n}$}

We start with the investigation of the simplest and most studied subset of $S_{n}$, i.e. with the set $P_{n}$ of periodic sequences. For the survey of the related literature we refer to $[2$.

As it is known, $\left(P_{n}, コ^{*}\right)$ is not a lattice for any $n \geq 2$, while $\left(P_{n}, \sqsupseteq\right)$ is a lattice for every $n \geq 2$ (cf. also Table 11). However, it is possible to prove something "positive" for $\sqsupseteq^{*}$ also in this case. Namely, we have

Theorem 1. Let $0 \leq \alpha \leq 1$, and put $H=\left\{A \in P_{2} \mid s_{2}(A)=\alpha\right\}$. Then $\left(H, \sqsupseteq^{*}\right)$ is a distributive lattice. 


\subsection{Ultimately Periodic Neighborhood Sequences $-U_{n}$}

As a natural extension of periodic sequences, now we consider ultimately periodic ones. Our first result shows that while $\left(P_{2}, コ^{*}\right)$ is not a lattice, the larger set $\left(U_{2}, コ^{*}\right)$ has this property.

Theorem 2. $\left(U_{2}, コ^{*}\right)$ is a non-complete distributive lattice.

However, it turns out that for larger values of $n$ the structure of $\left(U_{n}, コ^{*}\right)$ is not so nice, and we get

Theorem 3. $\left(U_{n}, コ^{*}\right)$ is not a lattice for $n \geq 3$.

The following result shows that the structural behavior of $U_{n}$ with respect to $\sqsupseteq$ is nice, just as in case of $P_{n}$.

Theorem 4. $\left(U_{n}, \sqsupseteq\right)$ is a non-complete distributive lattice for $n \geq 2$.

\subsection{Metrical Neighborhood Sequences $-M_{n}$}

From 3 we now that $\left(M_{2}, \sqsupseteq^{*}\right)$ forms a complete lattice, which is not distributive. However, this is the only "positive" result, as neither $\left(M_{n}, \beth^{*}\right)$ with $n \geq 3$, nor $\left(M_{n}, \sqsupseteq\right)$ with $n \geq 2$ are lattices. Here we provide the following related theorem. Note that by a result from [9], every $A \in M_{2}$ has density.

Theorem 5. Let $0 \leq \alpha \leq 1$, and set $H=\left\{A \in M_{2} \mid s_{2}(A)=\alpha\right\}$. Then $\left(H, コ^{*}\right)$ is a complete lattice.

Fazekas, Hajdu and Hajdu in [3] made the observation that for any $A, B \in M_{2}$ we have $A \wedge_{*} B \in M_{2}$, however, $A \vee_{*} B \in M_{2}$ does not always hold. As $\left(M_{2}, \beth^{*}\right)$ is a lattice, this implies that for any $A \in S_{2}$ there exists a uniquely determined $B \in M_{2}$ such that for any $C \in M_{2}$ with $C \beth^{*} A$ we have $C \beth^{*} B$. This $B$ is called the metrical closure of $A$, and is denoted by $M C(A)=B$. The authors in 3 derived several properties of the metrical closure, e.g. they provided an algorithm which generates $M C(A)$ for a fixed $A$. Now we prove the following result.

Theorem 6. For any $A \in P_{2}$ we have $M C(A) \in U_{2}$.

Note that by a simple example one can check that $A \in P_{2}$ does not necessarily implies $M C(A) \in P_{2}$. Further, unfortunately we are not able to prove (or disprove) the analogue of the above theorem for ultimately periodic sequences. So we formulate the following open problem:

Problem 1. Is it true that for any $A \in U_{2}$ we have $M C(A) \in U_{2}$ ? 


\subsection{Lyndon Sequences $-L_{n}$}

In this section we investigate the lattice properties of Lyndon sequences. This investigation is motivated by the simple but important observation that metrical neighborhood sequences are Lyndon sequences. Note that the opposite statement is false, as the Lyndon sequence $1112211211 \overline{2}$ is not a metrical neighborhood sequence. Unfortunately, it turns out that the set of Lyndon sequences does not form a nice structure neither under $\sqsupseteq^{*}$, nor with respect to $\sqsupseteq$.

Theorem 7. $\left(L_{n}, \sqsupseteq^{*}\right)$ and $\left(L_{n}, \sqsupseteq\right)$ are not lattices for any $n \geq 2$.

\subsection{Neighborhood Sequences Having Density $-D_{n}$}

It is obvious that sequences from $P_{n}$ and $U_{n}$ have densities. It is also known from [3] that the same holds for sequences from $M_{n}$. Hence it is natural to investigate general neighborhood sequences having density. In this section we give our results about the lattice structure of the set $D_{n}$ of such sequences.

Theorem 8. $\left(D_{2}, コ^{*}\right)$ is a non-complete distributive lattice.

Similarly to all the other investigated subsets of $S_{n}$, neither $D_{n}$ behaves nicely for higher dimensions with respect to $\sqsupseteq^{*}$.

Theorem 9. $\left(D_{n}, \sqsupseteq^{*}\right)$ is not a lattice for $n \geq 3$.

The following statement implies that $D_{2}$ has worse structural properties with respect to $\sqsupseteq$ than to $\sqsupseteq^{*}$.

Theorem 10. $\left(D_{n}, \sqsupseteq\right)$ is not a lattice for $n \geq 2$.

\subsection{Summarizing the Lattice Properties}

We summarize the lattice properties of the investigated subsets of $S_{n}$ under the orderings $\sqsupseteq^{*}$ and $\sqsupseteq$ in Table 1 . Though $\sqsupseteq^{*}$ shows a rather negative behavior from 3D on, this ordering describes better the relative spreading speed. Moreover $\sqsupseteq^{*}$ supersedes $\sqsupseteq$ in case of metrical expectations. The 3D space is also important from practical point of view, and this domain can be addressed by restricting the investigated subsets for a more successful comparison. Introducing a new ordering can be an alternative to overcome the negative results, as well. In this paper we focused only on the relations investigated in the literature so far.

\section{Auxiliary Results and Proofs of the Theorems}

In this section we give the proofs of our theorems. For this purpose we need some auxiliary results. Our first statement shows that if $A, B \in S_{2}$ have the same density tuples, and they belong to some special subset of $S_{2}$, then the same is true for $A \wedge_{*} B$ and $A \vee_{*} B$. More precisely, we have 
Table 1. Lattice properties of subsets of $S_{n}$ with respect to $\beth^{*}$ and $\sqsupseteq$

\begin{tabular}{|c||c|c|c||c||c|c|c|}
\hline \multicolumn{1}{|c||}{} & \multicolumn{3}{c||}{$\sqsupseteq^{*}(n=2)$} & $\sqsupseteq^{*}(n \geq 3)$ & \multicolumn{3}{c|}{$\sqsupseteq(n \geq 2)$} \\
\cline { 2 - 8 } & Lattice & Complete & Distributive & Lattice & Lattice & Complete & Distributive \\
\hline \hline$S_{n}$ & + & + & + & - & + & + & + \\
\hline$P_{n}$ & - & - & - & - & + & - & + \\
\hline$U_{n}$ & + & - & + & - & + & - & + \\
\hline$M_{n}$ & + & + & - & - & - & - & - \\
\hline$L_{n}$ & - & - & - & - & - & - & - \\
\hline$D_{n}$ & + & - & + & - & - & - & - \\
\hline
\end{tabular}

Lemma 1. Let $H \in\left\{P_{2}, U_{2}, D_{2}\right\}$ and let $A, B \in H$ with $s_{2}(A)=s_{2}(B)$. Then $A \wedge_{*} B, A \vee_{*} B \in H$.

Proof. Assume first that $H=P_{2}$, i.e. $A, B \in P_{2}$. Without loss of generality we may assume that $l$ is a common period length for both $A$ and $B$. As $s_{2}(A)=$ $s_{2}(B)$ we have $\mathbf{2}(A, l)=\mathbf{2}(B, l)$. Moreover,

$$
\mathbf{2}\left(A \wedge_{*} B, l\right)=\mathbf{2}\left(A \vee_{*} B, l\right)=\mathbf{2}(A, l)=\mathbf{2}(B, l)
$$

also hold. Hence for all $i \in\{1, \ldots, l\}$ we get that

$$
\left(A \wedge_{*} B\right)(i)=\left(A \wedge_{*} B\right)(i+l) \text { and }\left(A \vee_{*} B\right)(i)=\left(A \vee_{*} B\right)(i+l) \text {. }
$$

Thus $A \wedge_{*} B, A \vee_{*} B \in P_{2}$.

Let now $H=U_{2}$, that is $A, B \in U_{2}$. Without loss of generality we may assume that $A=A_{1} \overline{A_{2}}$ and $B=B_{1} \overline{B_{2}}$ with $c:=\left|A_{1}\right|=\left|B_{1}\right|$. Let $l$ be the least common multiple of $\left|A_{2}\right|$ and $\left|B_{2}\right|$. Then by $s_{2}(A)=s_{2}(B)$ we have $T:=\mathbf{2}(A, c, c+l)=$ $\mathbf{2}(B, c, c+l)$, and thus also $\mathbf{2}(A, c, c+k l)=\mathbf{2}(B, c, c+k l)=k T$ for any $k \in \mathbb{N}$. Hence we have

$$
\begin{gathered}
\mathbf{2}\left(A \vee_{*} B, c+k l+i\right)=\max \{\mathbf{2}(A, c+k l+i), \mathbf{2}(B, c+k l+i)\}= \\
\quad=\max \{\mathbf{2}(A, c+i), \mathbf{2}(B, c+i)\}+k T=\mathbf{2}\left(A \vee_{*} B, c+i\right)+k T
\end{gathered}
$$

for any $i=1, \ldots, l, k \in \mathbb{N}$. Consequently, $A \vee_{*} B \in U_{2}$. As a similar argument shows that $A \wedge_{*} B \in U_{2}$, the lemma is proved when $H=U_{2}$.

Finally, let $H=D_{2}$, so $A, B \in D_{2}$. As $s_{2}(A)=s_{2}(B)$ and

$$
\min \{\mathbf{2}(A, i), \mathbf{2}(B, i)\}=\mathbf{2}\left(A \wedge_{*} B, i\right) \leq \mathbf{2}\left(A \vee_{*} B, i\right)=\max \{\mathbf{2}(A, i), \mathbf{2}(B, i)\}
$$

for any $i \in \mathbb{N}$, the statement follows in this case, as well.

Proof (of Theorem 11). By Lemma1 for any $A, B \in P_{2}$ we have $A \vee_{*} B, A \wedge_{*} B \in$ $P_{2}$. As $\left(S_{2}, \sqsupseteq^{*}\right)$ is a distributive lattice, the theorem follows.

Lemma 2. Let $A, B \in D_{2}$ with $s_{2}(A)>s_{2}(B)$. Then there exists a $K \in \mathbb{N}$ such that $\left(A \wedge_{*} B\right)(i)=B(i)$ and $\left(A \vee_{*} B\right)(i)=A(i)$ for $i>K$. 
Proof. As $s_{2}(A)>s_{2}(B)$, there exists a $K \in \mathbb{N}$ such that for $i>K$ we have $\mathbf{2}(A, i)>\mathbf{2}(B, i)$. Hence the statement immediately follows.

Proof (of Theorem Q). Let $A, B \in U_{2}$. If $s_{2}(A)=s_{2}(B)$ then by Lemma 1 and if $s_{2}(A) \neq s_{2}(B)$ then by Lemma 2 we obtain that $A \wedge_{*} B, A \vee_{*} B \in U_{2}$. Hence as $\left(S_{2}, コ^{*}\right)$ is a distributive lattice, the same is true for $\left(U_{2}, コ^{*}\right)$. To prove that $\left(U_{2}, コ^{*}\right)$ is non-complete, put $A_{1}=\overline{12}$ and for $i \in \mathbb{N}$ let $A_{i+1}=\overline{A_{1} \ldots A_{i} 1^{i+1} 2}$. Writing $\mathcal{A}=\left\{A_{i} \mid i \in \mathbb{N}\right\}$, it is obvious that $A:=\bigwedge_{*} \mathcal{A}=121^{2} 21^{3} 21^{4} 2 \ldots$ does not belong to $U_{2}$. Suppose that there exists the greatest lower bound $B$ of $\mathcal{A}$ in $\left(U_{2}, コ^{*}\right)$. Then $A \sqsupseteq^{*} B$, hence from $s_{2}(A)=0$ we have $s_{2}(B)=0$ which implies that $B$ is of the form $B=B_{1} \overline{1}$. Put $k=\left|B_{1}\right|$, and consider the sequence $C=C_{1} \overline{1} \in U_{2}$ with $\left|C_{1}\right|=2 k+1$ and $C(j)=A(j)$ for $j=1, \ldots, 2 k+1$. Then $A \sqsupseteq^{*} C$ and from $\sum_{i=1}^{2 k+1} B(i)<\sum_{i=1}^{2 k+1} C(i)$ we derive the contradiction $B \unrhd^{*} C$. Hence the lattice $\left(U_{2}, \sqsupseteq^{*}\right)$ is not complete.

Proof (of Theorem [3). Fix $n \geq 3$ and put $A_{1}=\overline{31}, A_{2}=\overline{2}, B_{1}=2 \overline{13}$ and $B_{2}=13 \overline{1}$. Now for any $B_{3} \in U_{n}$ with $B_{3} \neq B_{1}, A_{1} \sqsupseteq^{*} B_{3}$ and $A_{2} \sqsupseteq^{*} B_{3}$ imply $B_{3} \nsupseteq^{*} B_{1}$. This means that if the greatest lower bound of $A_{1}$ and $A_{2}$ exists in $U_{n}$ then it must be $B_{1}$. However, $A_{1} \sqsupseteq^{*} B_{2}$ and $A_{2} \sqsupseteq^{*} B_{2}$ but $B_{1} \beth^{*} B_{2}$. This together with the fact that $A_{1}, A_{2}, B_{1}, B_{2} \in U_{n}$ imply that $A_{1}$ and $A_{2}$ have no greatest lower bound in $U_{n}$ with respect to $\sqsupseteq^{*}$ and the statement follows.

Proof (of Theorem 4). Let $A, B \in U_{n}$. Then we may write $A=A_{1} \overline{A_{2}}$ and $B=B_{1} \overline{B_{2}}$ with $\left|A_{1}\right|=\left|B_{1}\right|$ and $\left|A_{2}\right|=\left|B_{2}\right|$. Hence it is obvious that $A \vee B, A \wedge$ $B \in U_{n}$, and we get that $\left(U_{n}, \sqsupseteq\right)$ is a distributive lattice. To show that the lattice is non-complete, let $B_{i}=12^{i}(i \in \mathbb{N})$ and put $A_{k}=B_{1} \ldots B_{k} \overline{1}(k \in \mathbb{N})$ and $\mathcal{A}=\left\{A_{k} \mid k \in \mathbb{N}\right\}$. Suppose that $\mathcal{A}$ has a least upper bound in $U_{n}$ with respect to $\sqsupseteq$, which is given by $C=C_{1} \overline{C_{2}}$. Put $B=B_{1} B_{2} \ldots$, and observe that $B \in S_{n} \backslash U_{n}$. Hence, as obviously $\bigvee \mathcal{A}=B$, we have $C \sqsupseteq B$. Let $j$ be minimal with $C(j)=2$ and $B(j)=1$, and define $D \in U_{2}$ by

$$
D(l)= \begin{cases}B(l), & \text { for } 1 \leq l \leq j, \\ 2, & \text { for } l>j .\end{cases}
$$

Then clearly, $D \sqsupseteq A_{k}$ for all $k \in \mathbb{N}$, however, $D \sqsupseteq C$ does not hold. This shows that the lattice $\left(U_{n}, \sqsupseteq\right)$ is not complete.

Proof (of Theorem (5)). Let $L$ be an arbitrary subset of $M_{2}$ such that $s_{2}(A)=$ $s_{2}(B)=\alpha$ for all $A, B \in L$. Theorem 7 of [3] implies that $\bigwedge_{*} L \in M_{2}$. Define the sequence $A \in S_{2}$ in the following way. If $\alpha=1$ then put $A(1)=2$, otherwise let $A(1)=1$. Then if $A(i)$ is already defined, set $A(i+1)=2$ if $(i+1) \alpha \leq \mathbf{2}(A, i)+1$ and let $A(i+1)=1$ otherwise. By Lemma 2 from [9] we get that $A \in M_{2}$. Further, it is clear that $s_{2}(A)=\alpha$ and that $A \sqsupseteq^{*} B$ holds for any $B \in M_{2}$ with $s_{2}(B)=\alpha$. Let $C$ be the least upper bound of $L$ in $M_{2}$ with respect to $\beth^{*}$. Then we have $A \sqsupseteq^{*} C$, which immediately gives $s_{2}(C)=\alpha$, and the theorem follows. 
To prove Theorem 6 we need two lemmas. The first one gives a characterization of the metrical property of neighborhood sequences.

Lemma 3. Let $A \in S_{2}$. Then $A \in M_{2}$ if and only if for all $t \in \mathbb{N}, j \leq t$ and $k_{1}, \ldots, k_{j} \in \mathbb{N}$ with $\sum_{i=1}^{j} k_{i}=t$ we have $\mathbf{2}(A, t) \geq \sum_{i=1}^{j} \mathbf{2}\left(A, k_{i}\right)$.

Proof. First we show necessity. Let $A \in M_{2}$. For $t=1$ the statement is obvious. Let $T \in \mathbb{N}$, and assume that the condition is valid for all $t$ with $1 \leq t<T$. Further, let $k_{1}, \ldots, k_{j} \in \mathbb{N}$ with $\sum_{i=1}^{j} k_{i}=T$. Then as $A \in M_{2}$ we have

$$
\mathbf{2}(A, T) \geq \mathbf{2}\left(A, k_{1}\right)+\mathbf{2}\left(A, T-k_{1}\right) \geq \sum_{i=1}^{j} \mathbf{2}\left(A, k_{i}\right)
$$

and the necessity follows by induction. To prove sufficiency, take an $A \in S_{2}$ having the appropriate condition. Then for arbitrary $k_{1}, k_{2} \in \mathbb{N}$ with $k_{1}<k_{2}$ we have

$$
\mathbf{2}\left(A, k_{1}+k_{2}\right) \geq \mathbf{2}\left(A, k_{1}\right)+\mathbf{2}\left(A, k_{2}\right)
$$

which yields $\mathbf{2}\left(A, k_{1}, k_{1}+k_{2}\right) \geq \mathbf{2}\left(A, k_{2}\right)$. Hence $A \in M_{2}$, and the lemma follows.

The following lemma shows that when creating the metrical closure of a periodic sequence $A$, after a certain index $A$ "does not matter" any more, it is sufficient to take care of the metricity condition only.

Lemma 4. Let $A \in P_{2}$ having period length $l$ and $B \in M_{2}$. If $\mathbf{2}(A, t) \leq \mathbf{2}(B, t)$ holds for all $t=1, \ldots, l$ then $B \sqsupseteq^{*} A$.

Proof. Obviously, $\mathbf{2}(A, k) \leq \mathbf{2}(B, k)$ for $k=1, \ldots, l$. Assume that $k>l$ and write $k=q l+r$ with $q, r \in \mathbb{N}$, where $0 \leq r<l$. Now using our conditions and Lemma 3 we have

$$
\mathbf{2}(A, k)=q \mathbf{2}(A, l)+\mathbf{2}(A, r) \leq q \mathbf{2}(B, l)+\mathbf{2}(B, r) \leq \mathbf{2}(B, k),
$$

and the statement follows.

Proof (of Theorem [6). Let $A \in P_{2}$ with period length $l$. Put $B=M C(A)$ and denote by $r$ the minimal index with $1 \leq r \leq l$ for which $\frac{\mathbf{2}(B, r)}{r}=\max _{1 \leq i \leq l}\left\{\frac{\mathbf{2}(B, i)}{i}\right\}$.

Now we show that for an arbitrary $t \in \mathbb{N}$ with $t>l$ there exist non-negative integers $a_{1}, \ldots, a_{l}$ with $\sum_{i=1}^{l} a_{i} i=t$ such that $\mathbf{2}(B, t)=\sum_{i=1}^{l} a_{i} \mathbf{2}(B, i)$. Note that by Lemma 3 and Theorem 8 of [3] we get that $B(t)=2$ if and only if for some $k \in \mathbb{N}$ with $1 \leq k<t$ we have $\mathbf{2}(B, k)=\mathbf{2}(B, t-k, t-1)+1$. Hence $\mathbf{2}(B, l+1)=$ $\mathbf{2}(B, l)+\mathbf{2}(B, 1)$ if $B(l+1)=1$, and $\mathbf{2}(B, l+1)=\mathbf{2}(B, k)+\mathbf{2}(B, l+1-k)$ with the appropriate $k$ if $B(l+1)=2$. That is, our claim holds for $t=l+1$. 
Assume now that $T>l+1$, and that the property holds for every $t$ with $l<t<T$. Again, we have $\mathbf{2}(B, T)=\mathbf{2}(B, T-1)+\mathbf{2}(B, 1)$ if $B(T)=1$, and $\mathbf{2}(B, T)=\mathbf{2}(B, k)+\mathbf{2}(B, T-k)$ with the appropriate $k$ if $B(T)=2$. In both cases we get by the induction hypothesis that the property is valid for $T$, as well. Hence our claim follows. We call $a_{1}, \ldots, a_{l}$ a minimal combination system for $t$.

Let $u \in \mathbb{N}$ such that $u \geq u_{0}:=\sum_{i=1}^{l} r i$ and take a minimal combination system $a_{1}, \ldots, a_{l}$ for $u$. Then we have $a_{i} \geq r$ for some $i \in\{1, \ldots, l\}$. However, as $r \mathbf{2}(B, i) \leq i \mathbf{2}(B, r)$, we may assume that $a_{r}>0$. Let now $b_{1}, \ldots, b_{l}$ be a minimal combination system for $u+r$. Similarly as above, we may assume that $b_{r}>0$. Hence as

$$
\mathbf{2}(B, u)+\mathbf{2}(B, r) \leq \mathbf{2}(B, u+r)=\sum_{i=1}^{l} b_{i} \mathbf{2}(B, i)
$$

and by Lemma 3

$$
\mathbf{2}(B, u) \geq \sum_{i=1}^{l} b_{i} \mathbf{2}(B, i)-\mathbf{2}(B, r)
$$

holds, we get

$$
\mathbf{2}(B, u)+\mathbf{2}(B, r)=\mathbf{2}(B, u+r) .
$$

Note that the latter relation is valid for any $u \geq u_{0}$, in particular also if we write $u+1$ in place of $u$. This implies

$$
\begin{gathered}
\mathbf{2}(B, r)=\mathbf{2}(B, u, u+r)=\mathbf{2}(B, u+1, u+r)+\mathbf{2}(B, u, u+1), \\
\mathbf{2}(B, r)=\mathbf{2}(B, u+1, u+r+1)=\mathbf{2}(B, u+1, u+r)+\mathbf{2}(B, u+r, u+r+1) .
\end{gathered}
$$

Thus $\mathbf{2}(B, u, u+1)=\mathbf{2}(B, u+r, u+r+1)$, or in other words $B(u+1)=$ $B(u+r+1)$. This proves that $B \in U_{2}$, and the theorem follows.

Proof (of Theorem 7). To prove the statement in case of $\sqsupseteq^{*}$, we give a counterexample. Let

$$
A_{1}=112221122222211222121211 \overline{2}, \quad A_{2}=112221122221211222112222 \overline{2} .
$$

Then $A_{1}, A_{2} \in L_{n}$, and we have

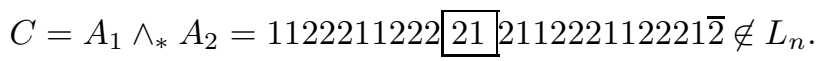

Moreover, let

$$
A = 1 1 2 2 2 1 1 2 2 2 \longdiv { 1 2 } 2 1 1 2 2 2 1 1 2 2 2 1 \overline { 2 } .
$$

Then $A \in L_{n}$. If $B \in L_{n}$ with $C \sqsupseteq^{*} B \sqsupseteq^{*} A$ then as only the eleventh and twelveth elements (boxed above) of $A$ and $C$ are different, we get that $A=B$. Thus we deduce that if the greatest lower bound of $A_{1}$ and $A_{2}$ in $L_{n}$ exists, then it must be $A$. However, for $D=111122222221211222112221 \overline{2} \in L_{n}$ we have $A_{1} \sqsupseteq^{*} D$ and $A_{2} \sqsupseteq^{*} D$, but $A \unrhd^{*} D$. This shows that $A$ cannot be the greatest lower bound of $A_{1}$ and $A_{2}$ in $L_{2}$. Thus $\left(L_{2}, \sqsupseteq^{*}\right)$ is not a lattice. 
Now we consider the ordering $\sqsupseteq$. Let $A=1222221 \overline{2}, B=1222122 \overline{2}$. As it can be easily seen, $A, B \in L_{n}$. We show that $A$ and $B$ do not have a greatest lower bound in $L_{n}$. Let $C=1212121 \overline{2}$ and $D=1122121 \overline{2}$. Clearly, $C, D \in L_{n}, A \sqsupseteq C$, $B \sqsupseteq C, A \sqsupseteq D$ and $B \sqsupseteq D$. Moreover, neither $C$ nor $D$ can be the greatest lower bound of $A$ and $B$ in $L_{n}$, since $C$ and $D$ cannot be compared. Looking at the first few elements of $A, B, C$ and $D$ we obtain that if the greatest lower bound $E$ of $A$ and $B$ in $L_{n}$ exists, then we must have $E=1222121 \ldots$. However, such a sequence cannot belong to $L_{n}$ and the statement follows.

Proof (of Theorem 8). Let $A, B \in D_{2}$. By Lemmas 1 and 2 it is obvious that $A \wedge_{*} B, A \vee_{*} B \in D_{2}$. Thus, as $S_{2}$ is a distributive lattice, $D_{2}$ is also a distributive lattice with respect to $\sqsupseteq^{*}$.

To prove that the lattice is not complete, let $A \in S_{2} \backslash D_{2}$ arbitrary with $\liminf _{k \rightarrow \infty} \frac{\mathbf{2}(A, k)}{k}=0$. For $i \in \mathbb{N}$ put

$$
A_{i}(j)= \begin{cases}A(j), & \text { for } j \leq i \\ 2, & \text { for } j>i\end{cases}
$$

and $P=\bigcup_{i=1}^{\infty} A_{i}$. Then as $A_{i} \in D_{2}$ with $s_{2}\left(A_{i}\right)=1$ for every $i \in \mathbb{N}$, we have $P \subseteq D_{2}$ and $\bigwedge_{*} P=A$.

Assume that $B \in D_{2}$ is the least upper bound of $P$ in $D_{2}$. Then as $A \sqsupseteq^{*} B$, $\frac{\mathbf{2}(A, k)}{k} \geq \frac{\mathbf{2}(B, k)}{k}$ must hold for all $k \in \mathbb{N}$ which by taking liminf yields $s_{2}(B)=0$.

Consider now the minimal index $t \in \mathbb{N}$ for which $A(t) \neq B(t)$. Then $A \sqsupseteq^{*} B$ implies $A(t)=2$ and $B(t)=1$. Put

$$
C(i)= \begin{cases}B(i), & \text { for } i<t \\ 2, & \text { for } i=t \\ 1, & \text { for } i>t\end{cases}
$$

Then $C \in D_{2}$ with $s_{2}(C)=0$, and we have $A \sqsupseteq^{*} C$. However, as $\mathbf{2}(C, t)>\mathbf{2}(B, t)$ we get $B \rrbracket^{*} C$ which contradicts the assumption that $B$ is the greatest lower bound of $P$ in $D_{2}$.

Proof (of Theorem 9). The validity of the statement can be easily checked by the same example as in the proof of Theorem 3. We omit the details.

Proof (of Theorem 10 ). Let $A=\overline{12}$ and let $B=V_{1} W_{1} V_{2} W_{2} V_{3} W_{3} \ldots$, where

$$
V_{k}=(12)^{2^{k-1}} \text { and } W_{k}=(21)^{2^{k-1}}
$$

for $k \in \mathbb{N}$. Note that $A, B \in D_{2}$ with $s_{2}(A)=s_{2}(B)=\frac{1}{2}$. For $C=A \vee B$ we have

$$
C=B_{1} 1^{2} B_{2} 1^{4} B_{3} 1^{8} B_{4} 1^{16} \ldots,
$$

where $B_{k}=(12)^{2^{k-1}}$ for $k \in \mathbb{N}$. It is easy to check that

$$
\liminf _{k \rightarrow \infty} \frac{\mathbf{2}(C, k)}{k}=\frac{1}{4} \text { and } \limsup _{k \rightarrow \infty} \frac{\mathbf{2}(C, k)}{k}=\frac{1}{3},
$$


whence $C \notin D_{2}$. Suppose that $D \in D_{2}$ is the greatest lower bound of $A$ and $B$ in $D_{2}$. Let $t \in \mathbb{N}$ be the minimal index such that $D(t) \neq C(t)$. Now $C \sqsupseteq D$ implies $D(t)=1$ and $C(t)=2$. For $i \in \mathbb{N}$ put

$$
E(i)= \begin{cases}C(i), & \text { if } i \leq t, \\ 1, & \text { if } i>t .\end{cases}
$$

Then we have $E \in D_{2}$ with $s_{2}(E)=0$, and $C \sqsupseteq E$. However, since $E(t)>D(t)$ we obtain $E \nsupseteq D$ which contradicts the assumption that $D$ is the greatest lower bound of $A$ and $B$ in $D_{2}$.

\section{References}

1. Das, P.P.: Lattice of octagonal distances in digital geometry. Pattern Recognition Lett. 11 (1990) 663-667

2. Fazekas, A., Hajdu, A., Hajdu, L.: Lattice of generalized neighbourhood sequences in $n \mathrm{D}$ and $\infty \mathrm{D}$. Publ. Math. Debrecen 60 (2002) 405-427

3. Fazekas, A., Hajdu, A., Hajdu, L.: Metrical neighborhood sequences in $\mathbb{Z}^{n}$. Pattern Recognition Letters 26 (2005) 2022-2032

4. Rosenfeld, A., Pfaltz, J.L.: Distance functions on digital pictures. Pattern Recognition 1 (1968) 33-61

5. Das, P.P., Chakrabarti, P.P., Chatterji, B.N.: Distance functions in digital geometry. Inform. Sci. 42 (1987) 113-136

6. Das, P.P.: Best simple octagonal distances in digital geometry. J. Approx. Theory 68 (1992) 155-174

7. Das, P.P., Chakrabarti, P.P., Chatterji, B.N.: Generalised distances in digital geometry. Inform. Sci. 42 (1987) 51-67

8. Fazekas, A.: Lattice of distances based on 3D-neighbourhood sequences. Acta Math. Acad. Paedagog. Nyházi. (N.S.) 15 (1999) 55-60

9. Hajdu, A., Hajdu, L.: Approximating the Euclidean distance by digital metrics. Discrete Math. 283 (2004) 101-111

10. Mukherjee, J., Das, P.P., Aswatha Kumar, M., Chatterji, B.N.: On approximating Euclidean metrics by digital distances in 2D and 3D. Pattern Recognition Lett. 21 (2000) 573-582

11. Hajdu, A., Hajdu, L., Tijdeman, R.: General neighbourhood sequences in $\mathbb{Z}^{n}$. Disc. Appl. Math. (to appear)

12. Lothaire, M.: Combinatorics on Words. Addison-Wesley, Reading, MA (1983) $\mathrm{xix}+238 \mathrm{pp}$.

13. Hajdu, A., Hajdu, L.: Velocity and distance of neighbourhood sequences. Acta Cybernet. 16 (2003) 133-145

14. Nivat, M.: Infinite words, infinite trees, infinite computations. In: Math Centre Tracts 109 (1979) 1-52

15. Siromoney, R., Mathew, R., Dare, V.R., Subramanian, K.G.: Infinite Lyndon words. Inf. Proc. Letters 50 (1994) 101-104

16. Yucas, J.L.: Counting special sets of binary Lyndon words. Ars Combinatoria 31 (1991) 21-29

17. Nagy, B.: Distance functions based on generalised neighbourhood sequences in finite and infinite dimensional spaces. In: E. Kovács, Z. Winkler (eds.),Fifth International Conference on Applied Informatics, Eger, Hungary, 2001, pp. 183-190

18. Nagy, B.: Distance functions based on neighbourhood sequences. Publ. Math. Debrecen 63 (2003) 483-493 